Revue d'histoire de l'Amérique française

\title{
De Charlevoix au Saguenay : caractéristiques des familles émigrantes au $\mathrm{XIX}^{\mathrm{e}}$ siècle
}

\section{Martine Hamel}

Volume 47, numéro 1, été 1993

URI : https://id.erudit.org/iderudit/305180ar

DOI : https://doi.org/10.7202/305180ar

Aller au sommaire du numéro

\section{Éditeur(s)}

Institut d'histoire de l'Amérique française

\section{ISSN}

0035-2357 (imprimé)

1492-1383 (numérique)

Découvrir la revue

\section{Citer cet article}

Hamel, M. (1993). De Charlevoix au Saguenay : caractéristiques des familles émigrantes au XIX ${ }^{\mathrm{e}}$ siècle. Revue d'histoire de l'Amérique française, 47(1), 5-25. https://doi.org/10.7202/305180ar

\section{Résumé de l'article}

Face au problème de saturation des terres qui se fait sentir dans Charlevoix au milieu du XIX ${ }^{\mathrm{e}}$ siècle, certaines familles vont opter pour l'émigration vers la périphérie. Ce mouvement migratoire va entraîner, dès les années 1830, la création d'une nouvelle région, le Saguenay. Notre étude propose une analyse en profondeur, à partir de Charlevoix, de variables socio-démographiques (âge, lieu de naissance, taille des ménages, nombre de garçons et de filles), et socio-économiques (profession, étendue des terres dans le cas des cultivateurs). Elle a permis d'établir un lien entre la situation des familles et leur propension à émigrer vers le Saguenay. Il est ressorti que ce sont les couples aux prises avec une charge familiale plus importante, et qui par le fait même occupaient de moins grandes superficies de terre par personne, qui ont opté pour l'émigration vers la région de colonisation. Cette migration répondait donc à un besoin, dans un contexte où il devenait de plus en plus difficile d'augmenter son capital foncier et de pourvoir à l'établissement des enfants. 


\title{
DE CHARLEVOIX AU SAGUENAY: CARACTÉRISTIQUES DES FAMILLES ÉMIGRANTES AU XIX ${ }^{\mathrm{e}}$ SIÈCLE ${ }^{1}$
}

\author{
MARTINE HAMEL \\ SOREP \\ Université du Québec à Chicoutimi
}

\section{RÉSUMÉ}

Face au problème de saturation des terres qui se fait sentir dans Charlevoix au milieu du XIX ${ }^{\mathrm{e}}$ siècle, certaines familles vont opter pour l'émigration vers la périphérie. Ce mouvement migratoire va entraîner, dès les années 1830 , la création d'une nouvelle région, le Saguenay. Notre étude propose une analyse en profondeur, à partir de Charlevoix, de variables socio-démographiques (âge, lieu de naissance, taille des ménages, nombre de garçons et de filles), et socio-économiques (profession, étendue des terres dans le cas des cultivateurs). Elle a permis d'établir un lien entre la situation des familles et leur propension à émigrer vers le Saguenay. Il est ressorti que ce sont les couples aux prises avec une charge familiale plus importante, et qui par le fait même occupaient de moins grandes superficies de terre par personne, qui ont opté pour l'émigration vers la région de colonisation. Cette migration répondait donc à un besoin, dans un contexte où il devenait de plus en plus difficile d'augmenter son capital foncier et de pourvoir à l'établissement des enfants.

\section{ABSTRACT}

Facing the problem of an increasing shortage of land, some families of the Charlevoix region in the mid-nineteenth century chose to migrate towards the periphery. As early as the 1830s, this movement led to the creation of a new region, the Saguenay. Taking Charlevoix as its starting point, a detailed analysis was conducted of socio-demographic variables (age, place of birth, household size, number of boys and girls), and socio-economic ones (profession, and, in the case of farmers, acreage owned). This exercice established a link between the families' situation and their propensity to migrate to the Saguenay. The study showed that larger families, which by dint of their size occupied smaller areas of land per person, chose to migrate towards the region of colonization. This migration thus met a need in a context where families found the tasks of increasing their holdings of land and "settling" their children more and more difficult.

1. Ce texte reprend quelques conclusions de mon mémoire de maîtrise (voir référence plus loin) déjà présentées au Congrès de l'Institut d'histoire de l'Amérique française à SaintJean-sur-Richelieu, en octobre 1990. Je tiens à remercier Danielle Gauvreau, ainsi que Gérard 


\section{INTRODUCTION}

Le mouvement migratoire à l'origine du peuplement de la région de Charlevoix débute à la fin du XVII ${ }^{\mathrm{e}}$ siècle, en provenance de la Côte-de-Beaupré. L'occupation du territoire s'effectue d'abord d'ouest en est sur les basses terres du littoral. Dès le début du XIX siècle la majeure partie de l'espace habitable est déjà occupée, et les bonnes terres du littoral se transigent à des coûts élevés (carte 1). La région de Charlevoix se trouve ainsi aux prises avec un problème de saturation de l'espace agraire. Plusieurs familles qui désirent agrandir la superficie de leur exploitation, notamment pour établir des enfants, se trouvent alors placées devant le choix de migrer vers les nouvelles paroisses en développement sur les plateaux de l'arrière-pays ou de quitter la région ${ }^{2}$. Il en résulte un processus migratoire complexe où les familles optent pour différentes stratégies, parfois même de façon simultanée ou successive. En effet les nouvelles zones de peuplement loin du littoral ne s'avèrent pas assez productives ${ }^{3}$. Les familles installées dans les nouvelles paroisses ont donc aussi recours à l'émigration, cette fois à l'extérieur de Charlevoix, pour résoudre le problème de disponibilité des bonnes terres. Ainsi, une étude réalisée par Michel Guérin démontre que les sous-régions de Charlevoix connaissent des bilans migratoires négatifs dès le milieu du $\mathrm{XIX}^{\mathrm{e}}$ siècle $^{4}$.

La présente étude concerne plus particulièrement un des mouvements migratoires privilégié par les Charlevoisiens, soit la migration de colonisation vers le Saguenay-Lac-Saint-Jean («Saguenay» dans la suite du texte). Ce déplacement de population fut en grande partie à l'origine du peuplement de la région du Saguenay. La proximité relative de ce territoire, alliée à l'exploitation forestière qui est amorçée depuis peu, font que les Charlevoisiens voient dans cette région une zone potentielle de colonisation. Le gouvernement du Canada ayant reconnu les possibilités agricoles de la région dès 1828 , des pressions sont exercées pour que le Saguenay soit officiellement

\footnotetext{
Bouchard, Raymond Roy et Marc St-Hilaire pour leurs commentaires. Cette étude fait partie d'un projet subventionné par le Fonds FCAR, réalisé à l'Université du Québec à Chicoutimi dans le cadre du Centre interuniversitaire de recherches sur les populations (SOREP).

2. À ce sujet, voir M.-A. Bluteau et S. Gauthier, «Eléments de recherche en vue d'une étude historique du peuplement de Charlevoix», Revue de la Société d'histoire de Charlevoix, 2,1 (1984): 6 .

3. Au sujet des paroisses de l'arrière-pays, voir R. Blanchard, L'est du Canada français, Province de Québec (Montréal, Beauchemin, 1935), 356.

4. M. Guérin, Peuplement et dynamique démographique de Charlevoix des origines à aujourd'hui, mémoire de maîtrise (Études régionales), Université du Québec à Chicoutimi, 1988, 249.
} 


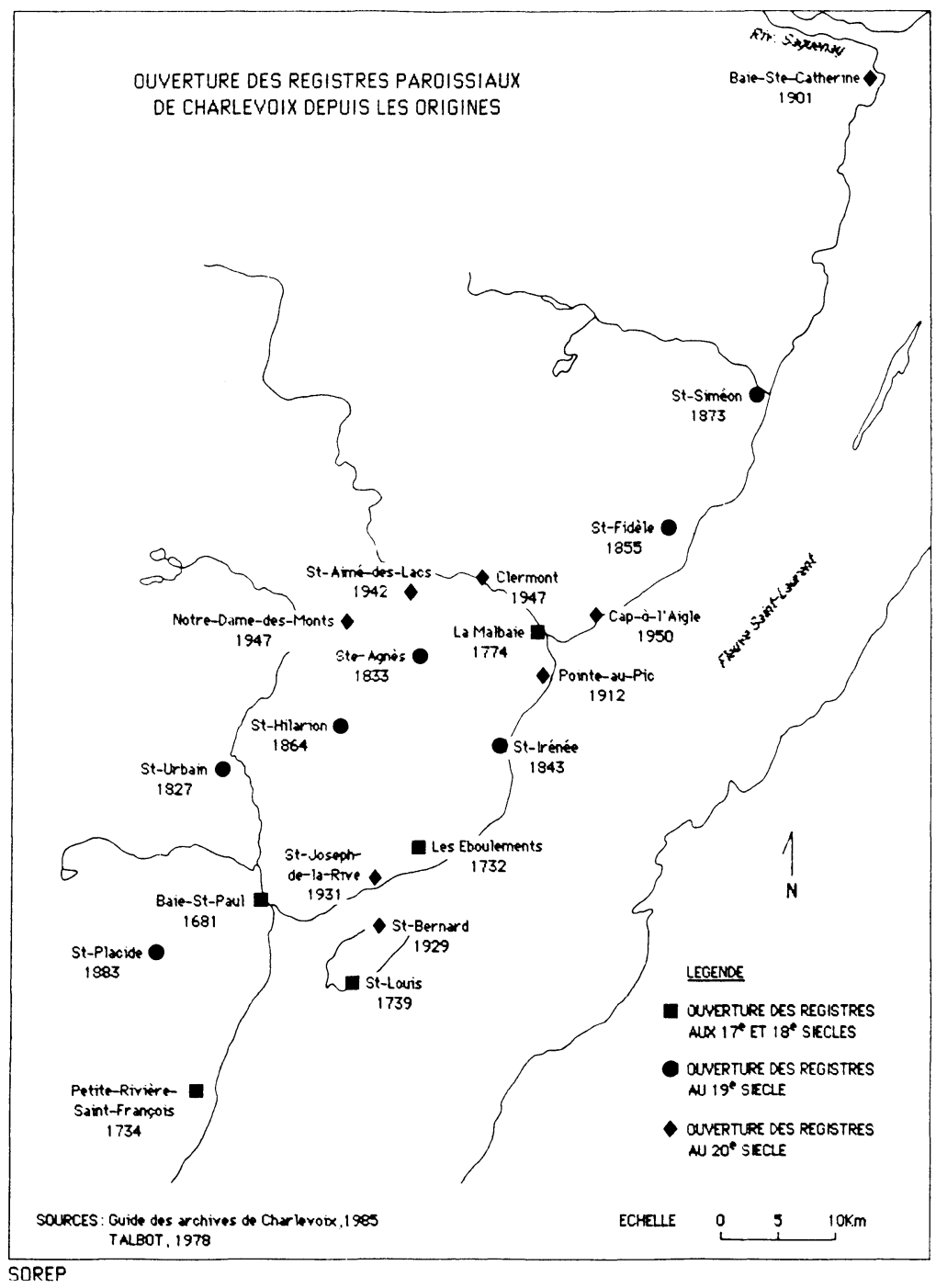

ouvert à la colonisation. À partir de ce moment, une pétition circule dans Charlevoix pour que le gouvernement permette l'établissement de colons au Saguenay. Mais la région étant la chasse gardée de la Compagnie de la Baie d'Hudson, le peuplement colonisateur ne débute vraiment qu'à la fin du bail de la compagnie en 1842. Dans certains cas l'émigration est saisonnière, les hommes laissant leur 
famille dans Charlevoix pour travailler tout l'hiver au Saguenay5. Bientôt ces migrations alternantes font place à une installation permanente.

Les immigrants en provenance de Charlevoix comptent pour $80 \%$ des personnes ayant émigré au Saguenay au cours des premières décennies. On peut, dans ce cas, parler de la création d'une région à partir d'une autre 6 . L'étude de ce mouvement à partir du lieu d'origine vise à faire ressortir les caractéristiques individuelles ou familiales des émigrants, contribuant ainsi à l'analyse des facteurs déterminants du processus migratoire. Elle constitue de plus un complément important aux travaux déjà effectués sur le même sujet à partir des sources saguenayennes (ils seront mentionnés plus loin).

\section{MIGRATIONS ET REPRODUCTION FAMILIALE}

La région de Charlevoix n'est pas la seule à connaître une importante mobilité géographique au cours de cette période. L'ampleur du phénomène migratoire dans plusieurs sociétés pré-industrielles du XIX ${ }^{\mathrm{e}}$ siècle est maintenant bien établie, et on sait qu'il a souvent impliqué des familles entières ${ }^{7}$. À la même époque, l'ensemble du Québec connaît d'importants mouvements de population, particulièrement marqués dans les régions rurales où les bilans migratoires sont négatifs depuis le milieu du $\mathrm{XIX}^{\mathrm{e}}$ siècle ${ }^{8}$. Le monde paysan réagit de diverses façons au problème de saturation des terres et aux difficultés qu'il entraîne. Certaines familles choisissent de quitter leur paroisse pour s'installer dans une autre nouvellement colonisée, d'autres optent pour la migration vers les villes en développement, au Québec et surtout aux États-Unis. Plusieurs études démontrent le caractère souvent familial de ces mouvements migratoires, qui

5. Sur l'ouverture du Saguenay à la colonisation, voir G. Bouchard, «Le peuplement blanc», C. Pouyez et al., Les Saguenayens. Introduction à l'histoire des populations du Saguenay, $16^{e}$ et $20^{e}$ siècles (Québec, Presses de l'Université du Québec, 1983); et J.-P.-M. Tremblay, «Cinq siècles d'histoire en Charlevoix», Saguenayensia, 26,2, numéro spécial, 1984, 84 p.

6. R. Roy, G. Bouchard et M. Declos, «La première génération de Saguenayens: provenance, apparentement, enracinement», Cahiers québécois de démographie, 17,1 (1988): 117.

7. À ce sujet, voir G. Darroch, «Migrants in the Nineteenth Century, Fugitives or Families in Motion?», Journal of Family History, 6,3 (1981): 257; D. Gagan, «Geographical and Social Mobility in Nineteenth Century Ontario: a Micro-Study", Canadian Review of Sociology and Anthropology, 13,2 (1976): 152; D. Parkerson, «How Mobile Were Nineteenth Century Americans?», Historical Methods, 15,3 (1982): 99; B. Ramirez, On the Move: French-Canadian and Italian Migrants in the North Atlantic Economy, 1860-1914 (Toronto, McClelland and Stuart, 1990), 172 p.; F. Gagnon, «Parenté et migration: le cas des Canadiens français à Montréal entre 1845-1875», Historical Papers/Communications historiques (1988): 63-85.

8. Voir C. Pouyez et al., op. cit., 249. 
paraissent ainsi s'inscrire dans la foulée de stratégies de reproduction familiale déjà bien ancrées dans les comportements.

L'émigration vers les centres urbains permet à plusieurs membres de la famille de trouver du travail. Dans le cas des chefs de famille qui cherchent plutôt à établir leurs enfants sur des terres, le modèle de transmission des avoirs familiaux privilégié est celui décrit par Gauldrée-Boileau à partir de son étude de la paroisse de Saint-Irénée? À mesure que les fils atteignent l'âge adulte, le père leur fournit les moyens d'acheter une terre dans une paroisse à proximité, de peuplement plus récent, et donc d'un coût moins onéreux. À partir du moment où il n'est pratiquement plus possible d'acheter des terres et que celles disponibles se transigent à des prix trop élevés, certaines familles optent pour l'émigration vers la périphérie, dans des territoires non défrichés ${ }^{10}$.

En émigrant vers une région de colonisation la famille peut espérer augmenter son capital foncier. La vente des terres possédées dans l'ancienne paroisse rend en effet possible l'achat de superficies beaucoup plus importantes dans les nouveaux territoires. Au Saguenay, Gérard Bouchard estime que la vente d'un lot dans une paroisse de peuplement ancien permet l'achat de superficies trois ou quatre fois plus étendues en région de colonisation ${ }^{11}$. Le père de famille de Charlevoix qui ne réussit pas à étendre son capital foncier peut donc se diriger vers les régions où la terre est encore disponible, et espérer être en mesure d'y établir ses enfants adultes ${ }^{12}$.

Des études réalisées sur les migrations de type familial au XIX ${ }^{\mathrm{e}}$ siècle suggèrent que toutes les familles ne sont pas touchées de la même façon par le phénomène, certaines variables pouvant avoir un effet plus ou moins déterminant sur la décision d'émigrer. Parmi les variables à caractère démographique, l'âge des chefs de ménage

9. C.-H.-P. Gauldrée-Boileau, «Paysan de Saint-Irénée», P. Savard, dir., Paysans et ouvriers québécois d'autrefois (Québec, Presses de l'Université Laval, 1968), 59-60.

10. Sur cette question, voir G. Bouchard et R. Thibeault, «L'économie agraire et la reproduction sociale dans les campagnes saguenayennes, 1853-1871", Histoire sociale/Social History, 18,36 (1986): 238; aussi, G. Bouchard, «Les modes de reproduction de la famille paysanne: en quoi, pourquoi la diversité? (Réflexion comparative des données québécoises et françaises)», Annales Économie Société Civilisation (à paraître).

11. G. Bouchard, «Démographie et société rurale au Saguenay, 1851-1935», Recherches sociographiques, 19,1 (1978): 27; et G. Bouchard, «Les migrations de réallocation comme stratégie de reproduction familiale en territoire neuf», Actes du Colloque d'histoire rurale comparée, Annecy, juin 1991 (à paraître).

12. Concernant l'établissement des fils adultes, voir D. Gagan, «Land, Population and Social Change: the 'Critical Years' in Rural Canada West», Canadian Historical Review, 59,3 (1978): 31; et H. J. Mays, «A Place to Stand: Families, Land and Permanence in Toronto Gore Township, 1820-1890», Canadian Historical Association (1980): 187. 
n'apparaît pas comme un facteur très important. C'est ce qui ressort de l'étude de Daniel Maisonneuve sur l'émigration à partir de la paroisse de Saint-Damase (comté de Saint-Hyacinthe) entre 1852 et 1861 , dans les cas où des familles entières émigrent ${ }^{13}$. Un phénomène semblable a été observé pour le Saguenay durant la même période, la relation établie entre l'âge des conjoints et la propension à émigrer étant assez faible ${ }^{14}$. Contrairement à ce qu'on aurait pu croire dans un premier temps, les enfants issus de familles nombreuses (en quelque sorte les surplus démographiques résultant de hauts niveaux de fécondité) ne sont pas les seuls touchés par l'émigration. D'une part, celle-ci implique souvent non seulement des individus isolés mais des familles entières et, d'autre part, la taille des familles n'apparaît pas comme une variable absolument déterminante dans le choix d'émi$\operatorname{grer}^{15}$. Toutefois, des études réalisées sur le Saguenay à l'échelle intrarégionale renforcent l'hypothèse d'un lien avec la composition des ménages, la sélection s'effectuant en faveur des ménages comptant plus de garçons que de filles ${ }^{16}$. Le nombre de garçons pèserait donc dans la décision d'émigrer, surtout dans le cas de migration vers des territoires de colonisation, lorsque l'établissement des enfants est en cause.

Pour Charlevoix la majorité des chefs de ménage migrant vers le Saguenay sont des cultivateurs, mais on note parmi eux une certaine surreprésentation des journaliers ${ }^{17}$, ce qui paraît correspondre à des stratégies visant à profiter des possibilités d'établissement foncier offertes par le nouveau territoire. Pour les propriétaires terriens certaines études établissent un rapport entre le nombre d'arpents de terre occupée et le fait de migrer ou non. Selon Daniel Maisonneuve, les ménages de Saint-Damase dont le nombre d'arpents de terre occupée est inférieur à 100 ont une plus forte propension à émigrer que ceux en possédant 100 et plus (soit 37\% d'émigrants contre 10\%). De la même façon, David Gagan dans son étude sur le comté de Peel (Ontario), estime que les familles avec des terres de 100 acres ou plus sont beaucoup plus sédentaires que celles qui en possèdent moins de $100^{18}$.

13. D. Maisonneuve, «Structure familiale et exode rural. Le cas de Saint-Damase, 18521861», Cahiers québécois de démographie, 14,2 (1983): 237.

14. C. Pouyez et al., op. cit., 145.

15. C. Pouyez et al., op. cit., 145; D. Maisonneuve, op. cit., 236.

16. G. Bouchard et J. Larouche, «Dynamique des populations locales: la formation des paroisses rurales au Saguenay (1840-1911)", Revue d'histoire de l'Amérique française, 41,3 (hiver 1988): 363-388; D. Gauvreau et M. Bourque, «Mouvements migratoires et familles: le peuplement du Saguenay avant 1911», RHAF, 42,2 (automne 1988): 167-192.

17. M. St-Hilaire, "Origines et destins des familles pionnières d'une paroisse saguenayenne au 19e siècle», Cahiers de géographie du Québec, 32,85, (1988): 11.

18. D. Maisonneuve, op. cit., 238; D. Gagan, Hopeful Travellers: Families, Land and Social Change in Mid-Victorian Peel County, Canada West (Toronto, University of Toronto Press, 1981), 120. 
La terre familiale peut suffire à faire vivre la famille sans cependant être suffisamment grande pour établir les enfants. Or, nous avons vu que la propension à émigrer des cultivateurs pouvait être liée non seulement au nombre d'arpents de terre occupée, mais aussi à la charge familiale. Ces deux variables, la taille du ménage et le nombre d'arpents de terre occupée, seraient donc indissociables, la situation économique du couple étant étroitement liée au nombre de personnes que la terre doit nourrir et au nombre d'enfants à établir. C'est une hypothèse que nous examinerons plus loin.

Avant de passer à l'analyse proprement dite, il est bon de se rappeler brièvement certains résultats de travaux sur la migration Charlevoix-Saguenay réalisés à partir des sources saguenayennes ${ }^{19}$. La prépondérance jusqu'en 1870 de la région de Charlevoix comme lieu d'origine des immigrants a été clairement démontrée. La principale caractéristique de ce phénomène migratoire est son caractère largement familial, particulièrement lorsque les immigrants proviennent de Charlevoix. La distribution par sexe des immigrants au Saguenay montre un rapport assez équilibré entre hommes et femmes au sein de la population adulte, des couples pour la plupart ${ }^{20}$. En effet, comme le démontrent les travaux de Danielle Gauvreau et Mario Bourque, il apparaît que les adultes célibataires ne représentent qu'une faible fraction des immigrants saguenayens avant $1912^{21}$. De plus, parmi les indicateurs qui font ressortir ce caractère familial de l'émigration, mentionnons la forte proportion des migrants arrivés alors qu'ils étaient enfants (62\%), c'est-à-dire accompagnés de leurs parents.

Jusqu'en 1911, la dimension moyenne du groupe familial (incluant père et mère, leurs frères et sœurs et les enfants le cas échéant) est de 6,8 personnes pour les immigrants provenant de Charlevoix ${ }^{22}$. Parmi le groupe des enfants immigrés au Saguenay, le rapport de masculinité est de 117,3 garçons pour 100 filles $^{23}$. Également présent dans des travaux sur la formation des paroisses rurales au Saguenay entre 1840 et 1911 , ce déséquilibre suggère que l'un des

19. Concernant les premiers immigrants au Saguenay, voir R. Roy et al., op. cit., 113134; M. St-Hilaire, op. cit., 5-26.

20. Voir R. Roy et al., op. cit., 118.

21. D. Gauvreau et M. Bourque, op. cit., 181.

22. D. Gauvreau et M. Bourque, «Les migrations interrégionales au Saguenay avant 1911», G. Bouchard, M. de Braekeleer et al., Histoire d'un génôme. Population et génétique dans l'est du Québec (Québec, Presses de l'Université du Québec, 1991), 187-212.

23. Concernant le rapport de masculinité au Saguenay, voir G. Bouchard et J. Larouche, op. cit., 387; D. Gauvreau et M. Bourque, op. cit., 180. 
motifs d'émigration réside dans la volonté d'établir des garçons, en même temps que ceux-ci constituent une main-d'œuvre importante pour les défrichements. Réalisée à partir du lieu de départ, l'analyse qui suit vise à compléter ce premier portrait du mouvement migratoire en fournissant des données plus précises sur la situation démographique et socio-économique des couples dans Charlevoix. Les caractéristiques spécifiques de ceux ayant émigré seront donc mises en évidence, et une mesure de l'émigration à partir de la région de Charlevoix sera proposée.

\section{PRÉSENTATION DES SOURCES ET DES ÉTAPES DE JUMELAGE ${ }^{24}$}

Pour des raisons surtout méthodologiques, l'étude porte sur la portion du contingent émigrant constitué par des couples et leurs enfants, lesquels représentent comme on l'a vu la majorité des immigrants saguenayens. Dans Charlevoix, ce type de ménage compte pour 95\% de l'ensemble, les autres ayant plutôt des personnes seules à leur tête (célibataires, veufs ou veuves), plus difficiles à identifier dans les sources disponibles.

Ainsi, la sous-population qui est l'objet de cette étude est constituée de 1193 couples recensés dans quatre paroisses de Charlevoix en 1852, soit Baie-Saint-Paul (497), La Malbaie (389), Sainte-Agnès (202) et Saint-Urbain (105). Ces paroisses représentent la moitié de celles dont les registres paroissiaux sont ouverts en 1852, et elles réunissent plus de $50 \%$ de la population présente dans Charlevoix au moment du recensement. On ne peut assurer que ces paroisses soient absolument représentatives de la situation vécue dans la région à cette époque. Cependant notre choix rend compte du clivage géographique (littoral-plateau) puisque ces quatre paroisses représentent tout aussi bien les paroisses anciennes du littoral, que les autres sises sur le plateau et plus récemment ouvertes à la colonisation.

Deux anciennes paroisses de peuplement du littoral, Baie-SaintPaul et La Malbaie, ont d'abord été choisies en raison de leur importance comme bassin d'émigration. Comme une étude de la première génération de Saguenayens l'a fait ressortir, un nombre élevé d'immigrants sont venus de ces deux paroisses pour s'établir au Saguenay; au cours des premières décennies, elles totalisent à elles seules $68 \%$ des lieux de mariage des pionniers, la paroisse de La Malbaie y étant du reste surreprésentée, surtout avant $1852^{25}$. En effet, comptant en 1844

24. Pour plus de précision, voir M. Hamel, L'émigration de Charlevoix vers le Saguenay au milieu du $19^{\circ}$ siècle: étude à partir du lieu d'origine, mémoire de maîtrise (Études régionales), Université du Québec à Chicoutimi, 1991, 166 p.

25. R. Roy et al., op. cit., 119. 
TABLEAU 1

Préparation des données

\begin{tabular}{|c|c|c|c|}
\hline \multicolumn{2}{|c|}{ Étapes, sources principales } & \multicolumn{2}{|c|}{ Buts, variables retenues } \\
\hline & $\begin{array}{l}\text { Recensement nominatif de } 1852 \\
\text { (Charlevoix) }\end{array}$ & $\begin{array}{l}\text { But: } \\
\text { Variables: }\end{array}$ & $\begin{array}{l}\text { identifier la population à l'étude dans les } \\
\text { quatre paroisses; recueillir les } \\
\text { informations la concernant } \\
\text { identification des personnes présentes } \\
\text { dans le ménage: âge, lieu de naissance, } \\
\text { profession }\end{array}$ \\
\hline $2-$ & Recensement agraire de 1852 (Charlevoix) & $\begin{array}{l}\text { But: } \\
\text { Variables: }\end{array}$ & $\begin{array}{l}\text { recueillir des informations de type socio- } \\
\text { économique concernant la population à } \\
\text { l'étude } \\
\text { nombre d'arpents de terre (occupée, en } \\
\text { culture, en pâturage), nombre de têtes de } \\
\text { bétail }\end{array}$ \\
\hline $3-$ & Registres paroissiaux (Charlevoix) & But: & $\begin{array}{l}\text { exclure de la recherche les couples } \\
\text { rompus par le décès d'au moins un } \\
\text { conjoint durant la période 1852-1861 }\end{array}$ \\
\hline & Fichier-réseau BALSAC de SOREP & $\begin{array}{l}\text { But: } \\
\text { Variables: }\end{array}$ & $\begin{array}{l}\text { vérifier si le couple à émigré au } \\
\text { Saguenay } \\
\text { numéro de la fiche de couple, lieu et date } \\
\text { de la première et de la dernière résidence, } \\
\text { la ou les professions exercées }\end{array}$ \\
\hline & $\begin{array}{l}\text { Recensement nominatif de } 1861 \\
\text { (Charlevoix) }\end{array}$ & But: & $\begin{array}{l}\text { retracer les couples qui résident encore en } \\
1861 \text { dans leur paroisse de résidence de } \\
1852\end{array}$ \\
\hline & $\begin{array}{l}\text { Recensement nominatif de } 1861 \\
\text { (Saguenay) }\end{array}$ & But: & $\begin{array}{l}\text { retracer les couples qui ont émigré au } \\
\text { Saguenay en } 1861\end{array}$ \\
\hline
\end{tabular}

pour le quart de sa région, la paroisse de La Malbaie est le lieu d'origine de $40 \%$ des pionniers saguenayens. Les paroisses de Saint-Urbain et de Sainte-Agnès, dont les registres s'ouvrent respectivement en 1827 et en 1833, sont largement issues des paroisses précédentes. Leur inclusion dans cette étude permettra de vérifier s'il existe une relation entre la propension à émigrer des couples et l'ancienneté du peuplement.

Notre analyse repose sur l'utilisation combinée de plusieurs sources. Pour la région de Charlevoix, les variables utilisées sont puisées dans les recensements nominatifs de 1852 et de 1861 , dans le recensement agraire de 1852 ainsi que dans les registres paroissiaux (sépultures). Le recensement nominatif de 1861 et le fichier de population SOREP sont utilisés pour le Saguenay. Les différentes étapes de préparation des données, présentées au tableau 1, ont conduit à une classification des couples en différentes catégories, selon leur cheminement entre 1852 et 1861 . 
Les deux premières étapes de la recherche ont consisté à dépouiller le recensement nominatif de 1852, puis à jumeler ces informations aux données du recensement agraire de la même année. L'ordre d'énumération étant généralement le même dans les deux recensements, il ne s'est pas posé de difficultés particulières lors du jumelage ${ }^{26}$. Les étapes subséquentes ont consisté à déterminer le destin des couples au cours de la période 1852-1861. On a cherché d'abord à repérer dans les registres paroissiaux de Charlevoix les sépultures enregistrées durant la période intercensitaire, de façon à exclure de l'étude les couples qui n'ont pas été soumis au risque d'émigrer durant toute la période. Les actes de sépultures n'ont fait l'objet d'une vérification que pour la paroisse concernée, c'est-à-dire celle dans laquelle le couple a été recensé en 1852 . Une vérification effectuée pour la paroisse de Baie-Saint-Paul a démontré que parmi les conjoints décédés, peu avaient été inhumés dans une autre paroisse. Quatrième étape, et la plus importante de cette recherche, le jumelage des couples restants avec le fichier-réseau BALSAC de $\operatorname{SOREP}^{27}$ a permis d'identifier les couples ayant émigré au Saguenay ${ }^{28}$. Rappelons que ce fichier repose sur les données des registres paroissiaux (1842-1871) et qu'il permet de suivre l'histoire démographique de chaque couple. Certains couples ont pu résider au Saguenay pendant une certaine période sans laisser de trace dans ce fichier (ou dans le recensement de 1861 utilisé dans l'étape suivante), mais la probabilité est faible, comme l'a démontré une étude ayant estimé le délai écoulé entre l'arrivée d'un couple au Saguenay et l'enregistrement d'un premier acte $^{29}$.

26. Sauf pour la paroisse de Saint-Urbain où l'ordre d'énumération était différent. Malgré tout, peu de couples étaient impliqués $(\mathrm{N}=105)$ et il a été possible de réaliser le jumelage en procédant par élimination. Le jumelage effectué peut être considéré comme aussi fiable que celui réalisé pour les autres paroisses.

27. G. Bouchard et al., Reconstitution automatique des familles. Le système SOREP (Chicoutimi, Université du Québec à Chicoutimi, SOREP, dossier n 2, 1979), 745 p., 2 volumes.

28. L'analyse ne concerne ici que les couples ayant immigré au cours de la période 18521861, mais le fichier permet aussi d'identifier ceux venus au Saguenay avant ou après cette période.

29. R. Jetté et D. Gauvreau, «Des fiches de famille à la mesure des migrations: une méthode élaborée à partir des données du Saguenay au XIX ${ }^{e}$ siècle», Cahiers québécois de démographie, 16,1 (1987): 37-65. 
TABLEAU 2

Catégorie migratoire des couples recensés dans quatre paroisses de Charlevoix en 1852, pour la période 1852-1861

\begin{tabular}{||l|c|c|c|c||c||}
\hline \multirow{3}{*}{\begin{tabular}{|l||c||} 
Catégorie \\
migratoire
\end{tabular}} & Baie-Saint-Paul & La Malbaie & Sainte-Agnès & Saint-Urbain & Ensemble \\
\cline { 2 - 6 } & & & & & \\
\hline Sédentaires & 74,7 & 67,2 & 56,8 & 69,4 & 68,6 \\
& $(307)$ & $(219)$ & $(100)$ & $(59)$ & $(685)$ \\
\hline Émigrants au & 10,2 & 13,8 & 11,9 & 15,3 & 12,1 \\
Saguenay & $(42)$ & $(45)$ & $(21)$ & $(13)$ & $(121)$ \\
\hline Autres émigrants & 15,1 & 19,0 & 31,3 & 15,3 & 19,3 \\
& $(62)$ & $(62)$ & $(55)$ & $(13)$ & $(192)$ \\
\hline \hline Ensemble & 100 & 100 & 100 & 100 & 100 \\
& $(411)$ & $(326)$ & $(176)$ & $(85)$ & $(998)$ \\
\hline
\end{tabular}

$X^{2}=26,12:$ significatif à 0,001

Sources: $\quad$ Recensements nominatifs de 1852 et 1861; Registres paroissiaux (sépultures); Fichier BALSAC (SOREP)

\section{MESURE ET CARACTÉRISTIQUES DU MOUVEMENT MIGRATOIRE}

Ampleur de l'émigration pour la période 1852-1861

Mentionnons d'abord que 16\% des 1193 couples de départ sont exclus de l'analyse en raison du décès de l'un des conjoints au cours de la période d'observation. Les autres couples sont regroupés selon leur statut migratoire durant la période 1852-1861: 1) sédentaires: ceux ayant déclaré le même lieu de résidence aux deux recensements; 2) émigrants au Saguenay entre 1852-1861 lorsqu'ils sont mentionnés comme résidents dans les registres paroissiaux saguenayens ou recensés au Saguenay en 1861;3) émigrants vers une autre destination (dits «autres émigrants») s'ils ne sont en $1861 \mathrm{ni}$ dans leur paroisse de résidence en 1852 ni au Saguenay. Dans ce dernier cas, l'émigration est survenue à l'intérieur ou encore vers l'extérieur de la région (excluant le Saguenay), les sources consultées ne permettant pas de faire la distinction entre les deux types. La catégorie la plus importante est celle constituée des couples sédentaires, c'est-à-dire ceux résidant dans la même paroisse aux deux recensements (tableau 2). Ces 685 couples $(68,6 \%)$ n'ont pas effectué de migration entre 1852 et 1861 , selon la définition retenue par cette enquête.

Plus de $30 \%$ des couples ont donc émigré, un résultat comparable à celui obtenu pour la paroisse de Saint-Damase étudiée par Daniel 
Maisonneuve $(32 \%)^{30}$. Près de $40 \%$ de ces couples ont émigré vers le Saguenay, soit un pourcentage plutôt élevé si on considère que ces couples se dirigeaient vers une région dont la colonisation n'avait débuté qu'une dizaine d'années plus tôt ${ }^{31}$. La dernière catégorie migratoire, composée de ceux que l'on ne retrace ni dans leur paroisse de résidence en $1852 \mathrm{ni}$ au Saguenay entre 1852 et 1861, regroupe 19,3\% de l'ensemble des couples. Il est probable qu'un fort pourcentage d'entre eux ont effectué un déplacement à l'intérieur de la région ${ }^{32}$.

Toutes les paroisses étudiées n'alimentent pas le mouvement migratoire vers le Saguenay de la même façon, mais toutes y versent entre $10 \%$ et $15 \%$ de leur population (tableau 2). Même les paroisses de peuplement récent ne parviennent pas à retenir leurs habitants, probablement parce que les terres disponibles ne sont pas de qualité suffisante. La petite paroisse de Sainte-Agnès, dont les registres paroissiaux ne s'ouvrent qu'en 1833, est représentative du phénomène: $40 \%$ des couples qui y sont recensés en 1852 ne s'y trouvent plus en 1861 .

\section{Caractéristiques des couples selon le statut migratoire ${ }^{33}$}

Les variables retenues pour l'analyse sont essentiellement celles disponibles dans le recensement de 1852 (tableau 1). En dépit de cette limite liée à la fois à la source et au fait que certaines variables ponctuelles ont pu changer entre 1852 et le moment de la migration, il a été possible de dresser un portrait démographique et socio-économique des couples. Ce portrait s'avère utile pour mettre en évidence d'éventuelles caractéristiques liées au statut migratoire. Dans la catégorie des variables démographiques on trouve l'âge et le lieu de naissance, deux variables individuelles, ainsi que la taille et la composition du ménage. La catégorie des variables socio-économiques comprend la profession de l'homme, disponible dans le recensement nominatif, ainsi que les variables du recensement agraire relatives à la superficie de terre occupée en culture et en pâturage, ainsi qu'au nombre de têtes de bétail.

30. D. Maisonneuve, op. cit., 234.

31. Il faut mentionner que le nombre de couples venus au Saguenay au cours de la période serait supérieur si on tenait compte des couples formés entre 1852-1861. Toutefois cela déborde le cadre de notre étude. En outre mentionnons qu'au moins un couple sur quatre, recensé dans Charlevoix en 1852, a émigré au Saguenay au cours de sa vie.

32. L. Boilard, Migrations internes dans la région de Charlevoix au milieu du $19^{\circ}$ siècle, mémoire de maîtrise (Études régionales), Université du Québec à Chicoutimi, 1991, 141 p.

33. La période intercensitaire étant de neuf ans, les couples qui ont émigré au Saguenay à la fin de la période, soit en 1861, peuvent présenter des caractéristiques qui different quelque peu de celles de 1852. 
TABLEAU 3

Âge des conjoints selon la catégorie migratoire

\begin{tabular}{|c|c|c|c|c|c|c|c|c|}
\hline \multirow[b]{2}{*}{ Groupe d'âges } & \multicolumn{2}{|c|}{ Sédentaires } & \multicolumn{2}{|c|}{$\begin{array}{c}\text { Émigrants au } \\
\text { Saguenay }\end{array}$} & \multicolumn{2}{|c|}{ Autres émigrants } & \multicolumn{2}{|c|}{ Ensemble } \\
\hline & Hommes & Femmes & Hommes & Femmes & Hommes & Femmes & Hommes & Femmes \\
\hline $\begin{array}{l}\text { Moins de } \\
30 \text { ans }\end{array}$ & $\begin{array}{r}24,7 \\
(169)\end{array}$ & $\begin{array}{r}38,9 \\
(266)\end{array}$ & $\begin{array}{l}22,3 \\
(27)\end{array}$ & $\begin{array}{l}28,1 \\
(34)\end{array}$ & $\begin{array}{l}28,6 \\
(55)\end{array}$ & $\begin{array}{l}33,4 \\
(64)\end{array}$ & $\begin{array}{l}25,2 \\
(251)\end{array}$ & $\begin{array}{l}36,5 \\
(364)\end{array}$ \\
\hline $30-39$ & $\begin{array}{r}27,7 \\
(189)\end{array}$ & $\begin{array}{l}27,0 \\
(185)\end{array}$ & $\begin{array}{l}23,1 \\
(28)\end{array}$ & $\begin{array}{l}38,8 \\
(47)\end{array}$ & $\begin{array}{l}21,4 \\
(41)\end{array}$ & $\begin{array}{l}25,5 \\
(49)\end{array}$ & $\begin{array}{l}25,9 \\
(258)\end{array}$ & $\begin{array}{l}28,2 \\
(281)\end{array}$ \\
\hline $40-49$ & $\begin{array}{r}24,9 \\
(170) \\
\end{array}$ & $\begin{array}{r}19,9 \\
(136) \\
\end{array}$ & $\begin{array}{l}28,9 \\
(35) \\
\end{array}$ & $\begin{array}{l}18,2 \\
(22) \\
\end{array}$ & $\begin{array}{l}21,4 \\
(41) \\
\end{array}$ & $\begin{array}{l}17,7 \\
(34) \\
\end{array}$ & $\begin{array}{l}24,7 \\
(246) \\
\end{array}$ & $\begin{array}{c}19,2 \\
(192) \\
\end{array}$ \\
\hline $50-59$ & $\begin{array}{r}16,4 \\
(112)\end{array}$ & $\begin{array}{l}10,4 \\
(71)\end{array}$ & $\begin{array}{l}23,3 \\
(27)\end{array}$ & $\begin{array}{l}14,0 \\
(17)\end{array}$ & $\begin{array}{l}15,6 \\
(30)\end{array}$ & $\begin{array}{l}15,1 \\
(29)\end{array}$ & $\begin{array}{l}17,0 \\
(169)\end{array}$ & $\begin{array}{l}11.7 \\
(117)\end{array}$ \\
\hline 60 ans et plus & $\begin{array}{l}6,3 \\
(43) \\
\end{array}$ & $\begin{array}{l}3,8 \\
(27) \\
\end{array}$ & $\begin{array}{l}3,4 \\
(4) \\
\end{array}$ & $\begin{array}{l}0,9 \\
(1)\end{array}$ & $\begin{array}{l}13,0 \\
(25) \\
\end{array}$ & $\begin{array}{r}8,3 \\
(16) \\
\end{array}$ & $\begin{array}{l}7,2 \\
(72) \\
\end{array}$ & $\begin{array}{r}4,4 \\
(44) \\
\end{array}$ \\
\hline Total & $\begin{array}{r}100 \% \\
(683)^{*}\end{array}$ & $\begin{array}{l}100 \% \\
(685)\end{array}$ & $\begin{array}{l}100 \% \\
(121)\end{array}$ & $\begin{array}{l}100 \% \\
(121)\end{array}$ & $\begin{array}{l}100 \% \\
(192)\end{array}$ & $\begin{array}{l}100 \% \\
(192)\end{array}$ & $\begin{array}{l}100 \% \\
(996)\end{array}$ & $\begin{array}{l}100 \% \\
(998)\end{array}$ \\
\hline Age moyen & 39,6 & 35,5 & 40,7 & 36,5 & 41,8 & 38,4 & 40,2 & 36,2 \\
\hline
\end{tabular}

* Deux indéterminés

Il est difficile d'établir un portrait type des couples d'émigrants au Saguenay sur la base de l'âge des conjoints. Ainsi, l'étalement de la distribution de l'âge des hommes et des femmes à la tête des couples d'émigrants au Saguenay indique bien qu'aucun groupe d'âges n'est épargné (tableau 3). On retrouve là, traduit d'une autre façon, le fait que le phénomène migratoire présente souvent un caractère familial élargi, impliquant autant des jeunes couples que d'autres formés depuis longtemps. Dans l'ensemble toutefois, les couples d'émigrants au Saguenay sont significativement un peu plus âgés que les couples sédentaires et un peu plus jeunes que les «autres émigrants». La différence tient surtout dans le premier cas aux nombreux couples où l'homme est dans la cinquantaine, ce qui est le fait de migrations liées à l'établissement des enfants. Les couples ayant émigré vers une autre destination sont proportionnellement plus nombreux aux deux extrêmes de la distribution, mais l'effet est plus marqué aux âges élevés, ce qui tient peut être au fait que la migration s'effectue plus rarement dans ce cas vers une région de colonisation.

L'analyse de leur déplacement jette un peu de lumière sur cette dernière situation (tableaux 4 et 5). La proportion de conjoints résidant en 1852 dans une paroisse autre que celle de leur naissance est la plus élevée parmi les couples ayant émigré vers une autre destination. Ce fait suggère que les conjoints plus âgés ont pu retourner dans leur lieu d'origine, surtout dans le cas où la naissance a eu lieu dans une autre région. Par contre, près des trois quarts des conjoints des autres catégories résidaient en 1852 dans la paroisse de leur naissance: on peut 


\section{TABLEAU 4}

Lieu de naissance des conjoints recensés dans quatre paroisses de Charlevoix en 1852, selon la catégorie migratoire

\begin{tabular}{||l|c|c|c||c||}
\hline \multirow{2}{*}{ Lieu de naissance } & \multicolumn{4}{|c||}{ Catégorie migratoire } \\
\cline { 2 - 5 } & \hline Sédentaires & $\begin{array}{c}\text { Émigrants vers } \\
\text { le Saguenay }\end{array}$ & $\begin{array}{c}\text { Autres } \\
\text { émigrants }\end{array}$ & Ensemble \\
\hline Même paroisse de résidence & 74,0 & 75,2 & 61,5 & 71,7 \\
qu'en 1852 & $(506)$ & $(91)$ & $(118)$ & $(715)$ \\
\hline Ailleurs dans Charlevoix & 20,9 & 20,7 & 26,0 & 21,9 \\
& $(143)$ & $(25)$ & $(50)$ & $(218)$ \\
\hline Extérieur de Charlevoix & 5,1 & 4,1 & 12,5 & 6,4 \\
& $(35)$ & $(5)$ & $(24)$ & $(64)$ \\
\hline \hline Ensemble & $100 \%$ & $100 \%$ & $100 \%$ & $100 \%$ \\
& $(684)$ & $(121)$ & $(192)$ & $(997)$ \\
\hline
\end{tabular}

\section{* Un indéterminé}

$X^{2}=19,25:$ significatif à 0,001

Source: Recensement nominatif de 1852

TABLEAU 5

Lieu de naissance des conjointes recensées dans quatre paroisses de Charlevoix en 1852, selon la catégorie migratoire

\begin{tabular}{||l|c|c|c||c||}
\hline \multirow{3}{*}{ Lieu de naissance } & \multicolumn{4}{|c|}{ Catégorie migratoire } \\
\cline { 2 - 5 } & Sédentaires & $\begin{array}{c}\text { Émigrants vers le } \\
\text { Saguenay }\end{array}$ & Autres émigrants & Ensemble \\
\hline Même paroisse de résidence & 72,8 & 72,8 & 60,9 & 70,6 \\
qu'en 1852 & $(499)$ & $(88)$ & $(115)$ & $(702)$ \\
\hline Ailleurs dans Charlevoix & 22,2 & 23,1 & 31,7 & 24,1 \\
& $(152)$ & $(28)$ & $(60)$ & $(240)$ \\
\hline Extérieur de Charlevoix & 5,0 & 4,1 & 7,4 & 5,3 \\
& $(34)$ & $(5)$ & $(14)$ & $(53)$ \\
\hline \hline Ensemble & $100 \%$ & $100 \%$ & $100 \%$ & $100 \%$ \\
& $(685)$ & $(121)$ & $(189)$ & $(995)$ \\
\hline
\end{tabular}

\footnotetext{
* Trois indéterminés
}

$X^{2}=10,65$ : significatif à 0,05

Source: Recensement nominatif de 1852 
TABLEAU 6

Distribution des conjoints suivant la catégorie socio-professionnelle et le statut migratoire

\begin{tabular}{|c|c|c|c|c|}
\hline \multirow[b]{2}{*}{ Catégorie migratoire } & \multicolumn{4}{|c|}{ Profession } \\
\hline & Cultivateurs & Journaliers & Autres & Ensemble \\
\hline Sédentaires & $\begin{array}{r}69,2 \\
(486)\end{array}$ & $\begin{array}{l}61,6 \\
(98)\end{array}$ & $\begin{array}{c}73,7 \\
(101)\end{array}$ & $\begin{array}{r}68,6 \\
(685)\end{array}$ \\
\hline Émigrants & $\begin{array}{c}30,8 \\
(216)\end{array}$ & $\begin{array}{l}38,4 \\
(61)\end{array}$ & $\begin{array}{l}26,3 \\
(36)\end{array}$ & $\begin{array}{c}31,4 \\
(313)\end{array}$ \\
\hline - vers le Saguenay & $\begin{array}{l}12,3 \\
(86)\end{array}$ & $\begin{array}{l}13,9 \\
(22)\end{array}$ & $\begin{array}{l}9,5 \\
(13)\end{array}$ & $\begin{array}{c}12,1 \\
(121)\end{array}$ \\
\hline - vers une autre destination & $\begin{array}{r}18,5 \\
(130) \\
\end{array}$ & $\begin{array}{l}24,5 \\
(39) \\
\end{array}$ & $\begin{array}{l}16,8 \\
(23) \\
\end{array}$ & $\begin{array}{r}19,3 \\
(192) \\
\end{array}$ \\
\hline Ensemble & $\begin{array}{l}100 \% \\
(702)\end{array}$ & $\begin{array}{l}100 \% \\
(159)\end{array}$ & $\begin{array}{l}100 \% \\
(137)\end{array}$ & $\begin{array}{l}100 \% \\
(998)\end{array}$ \\
\hline
\end{tabular}

$\mathrm{X}^{2}=5,77:$ significatif à 0,06

Source: Recensement nominatif de 1852

donc en déduire que les migrants vers le Saguenay en sont souvent à leur première expérience migratoire. Dans l'ensemble, la situation des femmes (tableau 5) diffère peu de celle des hommes (tableau 4), même si les hommes sont un peu plus nombreux à être nés à l'extérieur de Charlevoix. Cet écart découle du fait que les hommes effectuent davantage de migrations de longue distance que les femmes.

L'étude de la variable socio-professionnelle a été réalisée sur la base de trois grandes catégories: les cultivateurs, largement majoritaires avec 70\% des hommes à la tête des couples (702/998), les journaliers avec $16 \%$, et les autres professions regroupées en une seule catégorie avec 14\% (tableau 6). Tous les groupes sont touchés par le phénomène d'émigration mais, dans l'ordre, les émigrants sont proportionnellement plus nombreux parmi les journaliers et les cultivateurs. Ce phénomène est même plus marqué dans les jeunes paroisses du plateau, où la faible qualité des sols agricoles semble rendre encore moins facile l'établissement des couples sur une terre répondant aux besoins de la famille (données non présentées ici). À l'intérieur de chacune des catégories professionnelles, les émigrants optent à peu près également pour le Saguenay (un peu plus du tiers) ou pour une autre destination qui, rappelons-le, peut être la région de Charlevoix elle-même ou une autre région. 
TABLEAU 7

Nombre moyen d'enfants par famille selon le statut migratoire

\begin{tabular}{||l|c|c|c||c||}
\hline \multirow{2}{*}{ Nombre moyen d'enfants } & \multicolumn{3}{|c||}{ Catégorie migratoire } \\
\cline { 2 - 5 } & Sédentaires & $\begin{array}{c}\text { Émigrants vers } \\
\text { le Saguenay }\end{array}$ & Autres & Ensemble \\
\hline Nombre moyen de filles: & 1,38 & & 1,27 & 1,40 \\
- moins de 15 ans & 0,42 & 0,40 & 0,38 & 0,41 \\
- 15 ans et plus & 1,80 & 2,11 & 1,65 & 1,81 \\
- total & & & & 1,44 \\
Nombre moyen de garçons: & 1,43 & 1,81 & 1,22 & 0,49 \\
- moins de 15 ans & 0,49 & 0,64 & 0,40 & 1,93 \\
\hline - 15 ans et plus & 1,92 & 2,45 & 1,62 & 3,74 \\
\hline \hline $\begin{array}{l}\text { Nombre moyen d'enfants } \\
\text { par famille }\end{array}$ & 3,72 & 4,56 & 3,27 & $(998)$ \\
\hline
\end{tabular}

Source: Recensement nominatif de 1852

Ce portrait socio-démographique étant fixé, examinons maintenant la taille et la composition des ménages des différentes catégories migratoires, lesquelles seront mises en rapport dans un second temps avec les variables du recensement agraire. Les ménages considérés comprennent un couple à leur base, leurs enfants le cas échéant, et toutes les autres personnes, apparentées ou non, s'ajoutant à ce noyau ${ }^{34}$. Ces dernières font partie d'une catégorie appelée ici «autres personnes». Soulignons que le nombre d'enfants recensés ne représente évidemment pas la dimension totale ou définitive de la famille puisque, pour des raisons diverses, des enfants peuvent ne pas résider avec leurs parents lors du recensement. Il constitue cependant un bon indicateur de la charge familiale à un moment donné dans le temps.

Il ne semble pas y avoir de lien direct entre la dimension des ménages et la catégorie migratoire, la taille moyenne étant comparable pour chacune des catégories: 7,4 personnes par ménage pour les sédentaires, 7,6 pour les émigrants au Saguenay et 7,2 pour les «autres

34. Sans être parfaite, la reconnaissance des unités de résidence distinctes paraît assez fiable pour autoriser ce genre d'analyse basée sur les ménages. À ce sujet, voir N. Fortier, «Les recensements canadiens et l'étude de l'agriculture québécoise, 1852-1901», Histoire sociale/ Social History, 18,34 (1984): 257-286. 
émigrants» (tableau non reproduit ici). Le nombre moyen d'enfants est de 3,7 par ménage, soit 1,8 fille et 1,9 garçon (tableau 7). Ce nombre se répartit toutefois différemment selon les catégories, les émigrants au Saguenay comptant le plus grand nombre d'enfants dans le ménage, 4,6 en moyenne, comparativement à 3,7 et 3,3 respectivement pour les sédentaires et les autres. Rappelons que ces couples ayant émigré au Saguenay entre 1852 et 1861 se concentrent davantage dans les catégories d'âges où le nombre d'enfants présents dans le ménage est normalement plus élevé. Il n'en reste pas moins qu'ils ont à faire face à une charge familiale plus importante. La différence dans le nombre d'enfants se trouve compensée dans les autres groupes par les personnes s'ajoutant au ménage, leur répartition suivant l'âge établissant un équilibre assez semblable à celui des ménages ayant plus d'enfants.

Le nombre plus élevé d'enfants chez les émigrants au Saguenay est attribuable aux garçons et aux filles de moins de 15 ans, ainsi qu'aux garçons de 15 ans et plus. En effet, les ménages venus dans cette région comptaient en moyenne 1,7 fille et 1,8 garçon, contre 1,4 dans chacun des cas pour les couples sédentaires (différences statistiquement significatives). De plus, l'écart entre le nombre total de garçons et de filles est le plus élevé en faveur des garçons dans la catégorie des émigrants au Saguenay (2,4 garçons et 2,1 filles). Cet élément renforce l'idée que le nombre de garçons en âge de travailler aux défrichements et, bientôt de s'établir, peut influencer la décision d'émigrer, surtout lorsque cette dernière favorise des territoires de colonisation. Un résultat semblable ressort des travaux de E. A. Hammel et de son équipe pour les États-Unis. Ceux-ci suggèrent en effet que les familles avec un surplus de garçons tendent à s'établir plus souvent dans des régions à caractère rural ou en territoires de colonisation, tandis que celles comptant plus de filles choisissent des régions plus urbaines et plus industrialisées, en raison du travail rémunéré disponible pour les femmes ${ }^{35}$.

Comme il a été mentionné, certaines études ont établi l'existence, chez les cultivateurs, d'un rapport entre le nombre d'arpents de terre occupée et le fait de migrer ou non. Cette relation n'a cependant pas été observée ici. Il n'y a pas d'écart significatif au sein des cultivateurs selon le nombre d'arpents de terre occupée et la catégorie migratoire (tableau 8): $44 \%$ des cultivateurs ayant migré vers le Saguenay possèdent 100 arpents de terre ou moins, une proportion tout à fait semblable à celles obtenues pour les sédentaires (43\%) et les émi-

35. E. A. Hammel et al., «The Value of Children During Industrialization: Sex-Ratios in Childhood in Nineteenth-Century America», Journal of Family History (Hiver 1983): 351. 
TABLEAU 8

Distribution des cultivateurs suivant la catégorie migratoire et le nombre d'arpents de terre occupée

\begin{tabular}{|c|c|c|c|c|}
\hline \multirow[b]{2}{*}{ Nombre d'arpents } & \multicolumn{4}{|c|}{ Catégorie migratoire } \\
\hline & Sédentaires & $\begin{array}{c}\text { Émigrants vers le } \\
\text { Saguenay }\end{array}$ & $\begin{array}{c}\text { Émigrants vers } \\
\text { une autre } \\
\text { destination }\end{array}$ & $\begin{array}{c}\text { Ensemble des } \\
\text { émigrants }\end{array}$ \\
\hline $0-50$ & $\begin{array}{l}16,3 \\
(79)\end{array}$ & $\begin{array}{l}12,8 \\
(11) \\
\end{array}$ & $\begin{array}{l}17,7 \\
(23)\end{array}$ & $\begin{array}{l}15,7 \\
(34)\end{array}$ \\
\hline $51-100$ & $\begin{array}{l}26,3 \\
(128) \\
\end{array}$ & $\begin{array}{l}31,4 \\
(27) \\
\end{array}$ & $\begin{array}{l}31,5 \\
(41) \\
\end{array}$ & $\begin{array}{l}31,5 \\
(68) \\
\end{array}$ \\
\hline $101-150$ & $\begin{array}{l}24,9 \\
(121) \\
\end{array}$ & $\begin{array}{l}27,9 \\
(24) \\
\end{array}$ & $\begin{array}{l}20,8 \\
(27) \\
\end{array}$ & $\begin{array}{l}23,6 \\
(51) \\
\end{array}$ \\
\hline $151-200$ & $\begin{array}{l}17,1 \\
(83) \\
\end{array}$ & $\begin{array}{l}17,4 \\
(15) \\
\end{array}$ & $\begin{array}{l}15,4 \\
(20) \\
\end{array}$ & $\begin{array}{l}16,2 \\
(35) \\
\end{array}$ \\
\hline Plus de 200 & $\begin{array}{l}15,4 \\
(75) \\
\end{array}$ & $\begin{array}{c}10,5 \\
(9) \\
\end{array}$ & $\begin{array}{l}14,6 \\
(19) \\
\end{array}$ & $\begin{array}{l}13.0 \\
(29) \\
\end{array}$ \\
\hline Ensemble & $\begin{array}{l}100 \% \\
(486) \\
\end{array}$ & $\begin{array}{c}100 \% \\
(86)\end{array}$ & $\begin{array}{l}100 \% \\
(130) \\
\end{array}$ & $\begin{array}{l}100 \% \\
(216)\end{array}$ \\
\hline
\end{tabular}

$\mathrm{X}^{2}=4,8:$ non significatif à 0,1

Source: Recensement agraire de 1852

TABLEAU 9

Nombre moyen d'arpents de terre occupée par personne par famille, selon la paroisse et la catégorie migratoire des cultivateurs de Charlevoix en 1852

\begin{tabular}{|c|c|c|c|c|c|}
\hline \multirow[b]{2}{*}{$\begin{array}{l}\text { Catégorie } \\
\text { migratoire }\end{array}$} & \multicolumn{5}{|c|}{ Paroisse } \\
\hline & $\begin{array}{c}\text { Baie- } \\
\text { Saint-Paul } \\
\end{array}$ & La Malbaie & Sainte-Agnès & Saint-Urbain & Ensemble \\
\hline Sédentaires & $\begin{array}{r}27,9 \\
(199) \\
\end{array}$ & $\begin{array}{l}23,0 \\
(147) \\
\end{array}$ & $\begin{array}{l}25,0 \\
(94) \\
\end{array}$ & $\begin{array}{l}28,9 \\
(46) \\
\end{array}$ & $\begin{array}{r}25,9 \\
(486) \\
\end{array}$ \\
\hline Émigrants vers le Saguenay & $\begin{array}{l}24,7 \\
(28) \\
\end{array}$ & $\begin{array}{l}21,0 \\
(32) \\
\end{array}$ & $\begin{array}{l}15,9 \\
(16) \\
\end{array}$ & $\begin{array}{l}14,1 \\
(10) \\
\end{array}$ & $\begin{array}{l}20,4 \\
(86) \\
\end{array}$ \\
\hline Autres émigrants & $\begin{array}{l}38,3 \\
(38) \\
\end{array}$ & $\begin{array}{l}23,5 \\
(41) \\
\end{array}$ & $\begin{array}{l}20,0 \\
(44) \\
\end{array}$ & $\begin{array}{c}25,1 \\
(7) \\
\end{array}$ & $\begin{array}{r}26,7 \\
(130) \\
\end{array}$ \\
\hline Ensemble & $\begin{array}{l}29,0 \\
(265)\end{array}$ & $\begin{array}{l}22,8 \\
(220)\end{array}$ & $\begin{array}{r}22,7 \\
(154)\end{array}$ & $\begin{array}{l}26,1 \\
(63)\end{array}$ & $\begin{array}{l}25,4 \\
(702)\end{array}$ \\
\hline
\end{tabular}

Source: Recensement agraire de 1852 
grants vers d'autres destinations (49\%). Toutefois, le rapport entre cette superficie et le nombre de personnes par famille s'avère une variable plus déterminante. Évidemment restreinte aux cultivateurs (ils composent toutefois la majorité de la population), cette analyse fait ressortir une superficie moyenne plus faible de terre disponible par personne (parents et enfants présents dans le ménage) pour les familles de cultivateurs ayant émigré au Saguenay entre 1852 et 1861 (tableau 9). Dans l'ensemble, les autres catégories de sédentaires et d'émigrants vers d'autres destinations ont en commun une superficie moyenne par personne supérieure de près de 6 arpents. Ce résultat paraît d'autant plus significatif qu'il vaut pour chacune des paroisses prises séparément. Répétée avec d'autres indicateurs tels le nombre d'arpents de terre améliorée, cultivée ou en pâturage, ou encore le nombre de têtes de bétail, l'analyse conduit toujours au même résultat. Il ressort que la taille de la famille et la superficie de terre disponible sont des variables indissociables quand il s'agit de prendre la décision d'émigrer vers une région de colonisation. A une certaine surreprésentation des journaliers parmi les émigrants charlevoisiens, s'ajoute donc ici l'effet combiné de variables démographiques et économiques sur l'émigration des cultivateurs. Dans les deux cas, la difficulté de disposer de superficies suffisantes pour répondre adéquatement aux exigences de la reproduction familiale paraît à l'origine de stratégies familiales impliquant la migration. En arrière-plan de ces situations, on devine un processus encore mal connu au Québec, celui de l'élaboration de stratégies liées à des inégalités socio-économiques et démographiques.

\section{UN APERCUU DU DESTIN DES ÉMIGRANTS AU SAGUENAY}

L'analyse précédente, parce qu'elle porte sur une période restreinte et qu'elle est centrée sur les facteurs particuliers ayant pu influencer la migration, masque de bien des façons la complexité du processus migratoire et le caractère souvent non définitif des départs et des établissements. Mettant à profit le fichier-réseau BALSAC ainsi que le recensement de 1861, la partie qui suit cherche à enrichir cette vision très partielle du phénomène en examinant le destin des couples charlevoisiens ayant émigré au Saguenay entre 1852 et 1861.

L'analyse des fiches de famille suggère l'existence de stratégies migratoires complexes, faisant place à un nombre important d'aller et retour entre Charlevoix et le Saguenay, et témoignant souvent de plus d'une tentative de la part des familles avant que l'établissement ne soit définitif. À titre d'exemple, Pierre Bouchard et Marie Lavoie ${ }^{36}$, mariés

36. Il s'agit de noms fictifs. 
à La Malbaie en 1839, sont présents au Saguenay de 1847 à 1850, période au cours de laquelle le couple enregistre la naissance de deux enfants. Pierre Bouchard se déclare alors cultivateur. Le couple est ensuite de retour dans Charlevoix, où il est recensé à La Malbaie en 1852 dans le ménage des parents de l'époux. L'homme se déclare alors journalier et le couple compte quatre enfants. Ils sont de retour au Saguenay et y font baptiser un enfant en juillet 1852. Les deux conjoints décèdent dans cette région, d'où l'on peut conclure que le couple s'y est par la suite installé définitivement.

Parmi les 121 couples ayant émigré de Charlevoix vers le Saguenay entre 1852 et 1861 , près de $75 \%$ des conjoints y sont décédés, ce qui suggère qu'ils s'y sont établis définitivement. Comme des études l'ont déjà montré, cette proportion assez importante ne vaut probablement que pour cette région, puisque les couples charlevoisiens paraissent s'établir plus souvent définitivement au Saguenay que ceux en provenance d'autres régions $(\mathrm{N}=35)$. Il est probable que leur enracinement au Saguenay s'est trouvé facilité par les liens de parenté plus nombreux existant entre les nouveaux arrivants et ceux établis depuis quelque temps dans la région.

Les proportions précédentes ne varient guère suivant la paroisse d'origine dans Charlevoix. Au Saguenay, les couples établis dans la sous-région du Lac-Saint-Jean, peu nombreux il est vrai à cette époque, s'y installent presque tous définitivement. Au chapitre des différences, mentionnons que les couples plus jeunes quittent plus souvent le Saguenay que les autres. Cette situation est en partie normale puisqu'en raison de leur âge, ils sont plus souvent confrontés aux difficultés du premier établissement, avec de jeunes enfants qui ne peuvent participer aux travaux de défrichement. Par ailleurs, les couples où l'homme déclare exercer au Saguenay une occupation autre que cultivateur ou journalier sont ceux qui s'établissent le moins souvent définitivement. Ces hommes sont de petits industriels ou des commerçants dont la mobilité n'est pas restreinte par un enracinement à la terre et peut même être propice à la conclusion de nouvelles affaires. Soulignons enfin qu'une grande diversité caractérise aussi la situation des couples qui quittent le Saguenay. Leur durée de résidence dans la région, même mesurée imparfaitement (par le biais de la différence entre la date de la première et de la dernière mention du couple dans les registres saguenayens), varie entre 1 et 41 ans, la moyenne se situant autour d'une quinzaine d'années. 


\section{CONCLUSION}

L'étude à partir du principal lieu d'origine des premiers immigrants venus au Saguenay au milieu du XIX ${ }^{\mathrm{e}}$ siècle a d'abord permis de constater la ponction importante opérée sur la population de Charlevoix au cours de cette période. Près de $15 \%$ des couples recensés en 1852 émigrent en effet au Saguenay entre 1852 et 1861 , et près du quart d'entre eux le font à un moment ou l'autre de leur existence. Au-delà de ces proportions, les informations recueillies dans les sources saguenayennes sur les trajectoires souvent complexes des familles migrantes accentuent encore l'idée d'un brassage intense de populations qui émerge de plus en plus des études sur cette période du Québec rural.

Le phénomène est généralisé et aucune paroisse ou catégorie sociale n'y échappe. Pas plus les paroisses nouvellement ouvertes sur les plateaux que celles dont le peuplement est plus ancien; pas plus les couples d'âge moyen que les jeunes couples en voie d'établissement. C'est que les difficultés sont présentes dans tous les milieux, soit parce que les terres ne sont plus disponibles à des prix abordables ou que leur qualité fait problème. Dans tous les cas, la migration apparaît comme l'élément capital d'une stratégie qui vise à assurer l'établissement des couples et celui des descendants immédiats.

En plus du caractère intense du recours à l'émigration, cette étude menée à partir de la région d'origine a aussi révélé l'existence d'un certain nombre de facteurs associés à une plus grande propension à émigrer au Saguenay. Les plus importants concernent les familles comptant davantage de garçons, celles ayant à leur tête un journalier et, parmi les cultivateurs, les familles disposant d'une plus faible superficie de terre. Ces résultats suggèrent une implication étroite des facteurs d'ordre démographique et socio-économique inhérents à la logique de reproduction familiale. Celle-ci se déploie toutefois selon des modalités variées qu'il nous reste encore à approfondir. 